\title{
A manual for planetary management
}

It is time for environmental scientists and policy-makers to speak the same language, and to target the achievable, not simply the desirable. A framework is emerging from the International Geosphere-Biosphere Programme.

\section{Philip Newton}

When it comes to global environmental change, scientists and policy-makers rarely interact effectively. The reasons are not difficult to understand. Policy-makers prefer to make decisions, especially expensive ones, based on a degree of certainty, yet such is the elusive complexity of the Earth system that scientists are often able to do little more than outline a broad range of possibilities.

Witness the situation regarding future anthropogenic emissions of greenhouse gases: scientific advice on the likely impacts largely amounts to a wide range of possible consequences along with a warning that surprises are likely. It is hardly surprising that the largely politically brokered Kyoto Protocol amounts to little more than a tiny step towards limiting climate change ${ }^{1}$.

So how can scientists increase the chances of effective management of global change? Clearly there is a need to improve understanding of how the Earth system functions, but how can this be focused so as to optimize the role of policy-makers? Such questions were a strong motivation for the second congress of the International GeosphereBiosphere Programme (IGBP), held in $\mathrm{May}^{2}$.

The business of the IGBP is global-change research, which demands large-scale interdisciplinary actions. The IGBP's strategy over the past decade has been to coordinate activities within and between independently established international 'core' research programmes covering atmospheric chemistry, terrestrial and marine ecosystems, land-use change, hydrology, land-ocean interactions, ocean biogeochemistry and past climate change $^{2}$. This strategy has had successes. For example, as a result of such coordination it is now generally accepted (and widely touted) that the anticipated carbon sequestration by land vegetation in the coming century or so is likely to hit a ceiling. The identified limiting factors include the diminishing response of photosynthesis to an increasing burden of atmospheric $\mathrm{CO}_{2}$, a temperature-driven increase in respiration of plant carbon in soils, and, in systems where vegetation replacement is induced, the slow rate of carbon gain during growth relative to that during vegetation loss.

But, although many research communities have mixed and broadened constructively, such as between hydrological and landuse-change communities, there remains an urgent need to stretch further. For example, are physical and social scientists, or even terrestrial and marine ecologists, yet speaking the same language? And, if understanding the past is the key to the future, to quote the palaeoscientist's maxim, why has the integration of palaeoscience with other IGBP programmes been rather limited?

To tackle such issues, there is a need to map out a new strategy for global-change research. First, if the prerequisite of an improved understanding of the Earth system is to be attained, then research must become yet more interdisciplinary. Second, the focus needs to be on achievable and policy-relevant aims over the next decade - addressing questions we feel we can realistically answer, not those we would like to just out of curiosity. Third, a framework for integration of the observational, experimental and modelling activities is needed from the outset, to guide strategy. Leaving the integration until the end has done a fine job of showing where efforts should have been focused, when it is too late to fill the gaps. And, as Pam Matson of Stanford University puts it, funding across disciplines should be "matrix money, not glue money".

These are laudable aims, but what do they imply on a practical level? As a first step, most IGBP programmes have embarked on a phase of synthesis. This will elucidate the way forward - what do we know, what do we think we know, and what do we need to know, to achieve goals relevant to policy? Part of the 'need to know' challenge must include how to deal with upand down-scaling in space and time. This process is intended to spawn the programmes required to attain such goals.

It will be very hard to establish an integration framework, particularly on a global scale, as present capabilities for modelling the Earth system are rather limited. A dual approach is planned. On the one hand, the relatively conventional approach of improving coupled atmosphere-ocean-land-ice models will be pursued. Ingenuity aside, the computational demands are extreme, as is borne out by the Earth System Simulator 640 linked supercomputers providing 40 teraflops and a cooling system from hell under one roof - to be built in Japan by 2003.

The second course is the development of Earth-system analysis, such as proposed by John Schellnhuber ${ }^{3}$, as a hypothesis-testing framework. Such analyses aim to include the social dimension, ultimately aspiring to, as Schellnhuber puts it, a mathematical analysis of sustainable development, leading to the notion of a 'manual' for planetary management. A policy-relevant aim might be to develop such models to identify the likely pathways of human activities that could lead to intolerable risks to the global environment, so as to chart probable safe courses.

Integration on local to regional scales for problem-solving studies is somewhat less daunting. But if a region undergoing environmental change is to be investigated, the strategy from the outset should involve scientists across all the relevant disciplines, from the physical to the social, from ecology to economics. Such an approach is exemplified by the Large-scale Biosphere-Atmosphere experiment in Amazonia ${ }^{4}$, which embraces many IGBP core programmes.

A framework for future studies is emerging. The emphasis would be on environmental change of relevance to society, at global and regional (but not national, to lessen political issues) scales, largely focused on the next 10-20 years, and linking the 'hard' sciences to the social. Centred on the

Earth system as a whole, crosscutting activities would target themes such as the carbon and water cycles, or ecosystem services. New activities at existing disciplinary interfaces would be encouraged. Plans are in place to improve crucially needed links to other international organizations such as the World Climate Research Programme, the International Human Dimensions Programme and the Food and Agriculture Organization.

This is an ambitious enterprise, and whether it stands or falls rests on not only the skill, but the enthusiasm, of the scientists involved. Crossing disciplinary barriers requires considerable effort, both intellectually and in procuring funds from organizations that are largely structured to operate by discipline. The IGBP has no crock of gold to back up its fine words. Moreover, much of the measurement and observational work will have less allure than curiosity-driven research. The motivational role of the IGBP should not be underestimated.

Philip Newton is a senior editor at Nature and a visiting research fellow at the School of Biological and Molecular Sciences, Oxford Brookes University, Oxford OX2 OBP, UK.

\footnotetext{
1. Parry, M. et al. Nature 395, 741 (1998).

2. http://www.igbp.kva.se

. Schellnhuber, H.-J. \& Wenzel,V. (eds) Earth System Analysis: Integrating Science for Sustainability (Springer, Berlin, 1998).
} 4. http://www.cptec.inpe.br/lba 